\title{
Kernos
}

Revue internationale et pluridisciplinaire de religion grecque antique

$10 \mid 1997$

Varia

\section{Religion poliade et sectes}

\section{Pierre Lévêque}

Édition électronique

DOI : $10.4000 /$ kernos.662

ISSN : 2034-7871

\section{Éditeur}

Centre international d'étude de la religion grecque antique

\section{Édition imprimée}

Date de publication : 1 janvier 1997

Pagination : 233-240

ISSN : 0776-3824

\section{Référence électronique}

Pierre Lévêque, "Religion poliade et sectes », Kernos [En ligne], 10 | 1997, mis en ligne le 12 avril 2011, consulté le 02 mai 2019. URL : http://journals.openedition.org/kernos/662 ; DOI : 10.4000/kernos.662 


\section{Religion poliade et sectes}

La religion poliade est faite de cérémonies réglées, sous l'autorité des magistrats, citoyens délégués dans une charge et qui n'ont besoin d'aucune formation spéciale (sauf cas exceptionnels comme les mystères). Elle est une force de la cité, donnant élan aux grandes créations artistiques et littéraires, générant un consensus au moins momentané grâce aux festivités où s'assemblent dans la liesse tous les citoyens : les Panathénées de Phidias au Parthénon offrent au symbolique un bon exemple de son importance dans le fonctionnement de la polis classique, où le budget des cultes pèse au reste d'un bon poids. On se tromperait en affirmant qu'il s'agit d'une religion de faible religiosité. Des mythes d'une richesse exubérante fournissent l'arrière-plan aux formes si diverses du culte, comme la tragédie, où les exploits des temps héroïques évoquent le passé pré-civique et sont ainsi facteurs décisifs dans la quête d'identité collective. Plus adaptés aux besoins des couches populaires, les cultes à mystères apportent des révélations sur les dieux et leurs demeures, et des espérances pour une survie bienheureuse. L'équilibre qui s'est peu à peu créé à Athènes entre les anciens cultes poliades (celui de la sainte patronne Athéna notamment) et les cultes chthoniens des «deux déesses » ou de Dionysos enrichit la charge religieuse dont chacun peut faire son profit. Au reste, certaines liturgies qui ne sont point mystériques sont aussi l'objet de dévotions dont les péripéties étonnent, comme à Brauron où de petites filles de dix ans «font l'ourse » pour honorer Artémis et pour susciter les forces de fécondité en favorisant les passages pré-pubertaires.

Toutefois, les citoyens sont en manque de discours et de pratiques qui les fassent mieux pénétrer dans le monde des dieux... Ils voudraient une piété plus individuelle, résultant d'un choix, plus exaltante, et c'est un lieu commun de dire qu'à partir de 430 , les cultes étrangers ouvrent sur de nouvelles perspectives, établissent entre hommes et dieux des liens qui ne passent pas nécessairement par la médiation appauvrissante de la cité. Le processus qui nous retient ici est différent, mais il va dans le même sens : les communautés des hommes qui se sentent et se veulent frères et sours, qui pratiquent ensemble des cultes plus émotionnels. Les sectateurs de Perséphone, d'Orphée, de Dionysos pèsent d'un poids nouveau dans l'idéal des citoyens. Le tout ne se fait pas sans syncrétismes, c'est-à-dire sans confusion, d'où la nécessité d'une tentative de systématisation. 


\section{Les lamelles funéraires}

La riche documentation des lamelles d'or retrouvées dans des tombes permet d'aborder le thème des sectes. Leur diffusion est large et on les trouve avec une densité importante en Grande-Grèce, en Crète et dans la Grèce septentrionale. Le message change de l'une à l'autre, et il n'y a donc pas de texte canonique, mais en gros elles participent de quelques thèmes constants : le voyage aux Enfers dont il faut connaître la topographie, sous peine de se retrouver dans le secteur des damnés; le cyprès, la source qui rafraîchit ces assoiffés que sont les morts; les mots de passe théologiques:

- Je suis fils de la Terre et du Ciel étoilé.

- D'homme, tu es devenu dieu.

- Chevreau, tu es tombé dans le lait.

Une seule divinité est mentionnée : Perséphone.

Il vaut la peine de se pencher sur les tablettes récemment publiées de Pélinna (Thessalie), qui enrichissent notablement notre documentation ${ }^{1}$. Un prêtre (selon toute vraisemblance) interpelle le mort (la morte) dans ce moment décisif où il passe à une autre vie, par un véritable makarismos. Il devient "trois fois olbios » (ligne 1) et entre dans la catégorie des autres olbioi (l. 1 du a). Ce passage à un autre monde s'opère par le rituel du lait qui est ici dédoublé : « taureau, tu as jailli dans le lait ( nous aurons à le retrouver avec sa charge d'élan et de sexualité; « chevreau, tu es

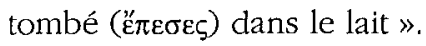

Loin de moi l'espoir de donner sens à cette image pastorale ${ }^{2}$ qui représente sans doute un rite d'initiation permettant de rompre avec la vie apparente de ce monde pour accéder à l'éternité.

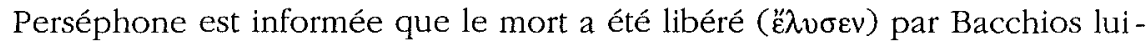

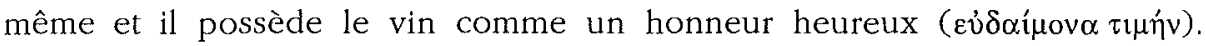
Question importante : pourquoi Dionysos est-il présent dans ce makarismos dominé religieusement par Perséphone ? Claude Calame le dit excellemment en notant que «autant la mention de Dionysos par son épiclèse Bacchios que l'olbos promis par cette forme énonciative particulière réfèrent le document de Pélinna à des mystères de type éleusinien ». Cette adjonction par rapport aux

1 Pour toutes les questions soulevées dans cet article, on se reportera à la bibliographie de l'article fondamental de $\mathrm{Cl}$. CALAME, Invocations et commentaires "orphiques": transpositions funéraires du discours religieux, in Discours religieux dans l'Antiquité. Actes du Colloque de Besançon, janvier 1995, Paris, 1995, p. 11 sq.

2 Sur ces expressions, peut-être faites pour garder un caractère secret, et sur la «zoologie dionysiaque de l'Outre-tombe », cf. la synthèse de G. CASADIO, Dioniso Italiota, in AION(archeol), 1994, p. 93-94. 
premiers textes pourrait être en relation avec l'emprise nouvelle du jeune dieu sur les cérémonies des mystères.

Dans les tablettes, la vie apparaît comme une préparation à l'olbos d'après la mort. C'est un message simple, en partie topographique, en partie théologique, formule au reste trop réductrice, car chacun des éléments du parcours infernal a un énorme poids anthropologique : Mémoire et Oubli, les cyprès, la source d'eau fraîche, seule capable de rassasier ces assoiffés, pour lesquels il n'est pas interdit de penser au terme mycénien di-pi-si-jo (de $\delta$ í $\psi \alpha$, «la soif », mot d'étymologie inconnue), selon l'heureuse hypothèse de L. Palmer. Sur le voyage lui-même, Circé donne déjà des informations concordantes à Ulysse $(O d ., \mathrm{X}, 510)$ : « Audelà du petit promontoire est le bois de Perséphone, avec ses saules et ses hauts peupliers; c'est ici qu'il faut échouer le navire ». Même si les arbres sont d'espèces différentes, on trouve déjà la même connotation du trajet vers les Enfers et du bois sacré où règne Perséphone.

En dehors de ces éléments paysagers, il est capital d'insister sur les éléments généalogiques (nous pouvons être rachetés en tant que fils de Terre et de Ciel) et sur les éléments moraux, pour lesquels on se reportera notamment aux tablettes de Thourioi où la pureté, la justice, la sainteté sont les maîtres-mots. L'initiation débouche en effet sur l'olbos, un vocable non grec d'étymologie, et dont le sens n'a cessé de se charger de religiosité mystérique ${ }^{3}$, qui se complète par un mot cette fois d'origine claire, eudaimon, appliqué au vin.

Que l'atmosphère soit éleusinienne n'est pas contesté; qu'on soit dans une forme de pensée marginale par rapport à celle des mystères officiels, intégrés depuis des siècles dans les cultes d'État, ne l'est pas davantage. D'abord seule est présente Perséphone et ce passage du duel au singulier est capital : nous avons non plus le couple primordial mère/fille (accompagné de l'enfant divin), mais seulement la déesse-fille, toujours invoquée ici sous son nom crétois de Perséphone, dispensatrice de l'olbos. Pourquoi cette déesse du salut, qui bien sûr n'est pas isolée par rapport à Éleusis ( $c f$. les personnages d'Eubouleus et d'Euclès dans une lamelle de Thourioi) ? Parce qu'elle est la reine infernale, celle qui réside partiellement en bas et que l'on va retrouver en descendant vers l'Hadès. Nous voici donc en présence d'une conception plus infernale du salut, dont par essence est exclue Déméter.

À cette riche documentation des lamelles on peut ajouter des cas de représentations graphiques non scripturaires qui semblent se substituer au texte dans des zones d'Italie où l'écriture ne s'était pas encore diffusée. Un bon exemple est fourni par un vase funéraire de Ripacandida (Basilicate) de la première moitié du $\mathrm{v}^{\mathrm{e}}$ siècle $^{4}$ (Fig. 1 = Tagliente, fig. 3). A l'intérieur d'un décor géométrique se profilent deux cercles concentriques : sur le plus grand un

\footnotetext{
3 Cf. mon article, Olbios et la félicité des initiés, in Rayonnement grec, Bruxelles, 1992, p. 113 sq.

4 M. TAGLIENTE, Ceramiche figurate nel mondo indigeno della Basilicata, in C. GELAO (éd.), Studi in onore di Micbele d'Elia, Matera, 1996, p. 39 sq.
} 
personnage et sept étoiles; au centre une sphère avec le foudre. Fabrication indigène dans une zone où la foi dans les réalités substantielles et transcendantes est vive. Le personnage, complètement disproportionné, lève ses énormes mains dans un geste de deuil ou de supplication; le cercle extérieur est le ciel (avec ses sept astres figurant le soleil, la lune et les cinq planètes alors connues, plutôt que la Grande Ourse); le cercle intérieur est la terre, avec le foudre de Zeus. C'est ce qu'expriment les lamelles en subordonnant le salut du mort à son insertion dans le cosmos, Ciel et Terre : «je suis par les Titans fils de Terre et du Ciel étoilé, mais ma race est céleste », avec la présence symbolique de Zeus représenté par son foudre, organe de liaison entre Ciel et Terre.

L'interprétation est confirmée par le fait qu'on retrouve le même ensemble sur un denier d'or frappé par l'empereur Domitien pour déplorer la mort de sa fille Domitia, à cette différence près que le personnage de Ripacandida est remplacé par un enfant assis sur le globe terrestre (Fig. 2 = Tagliente, fig. 4).

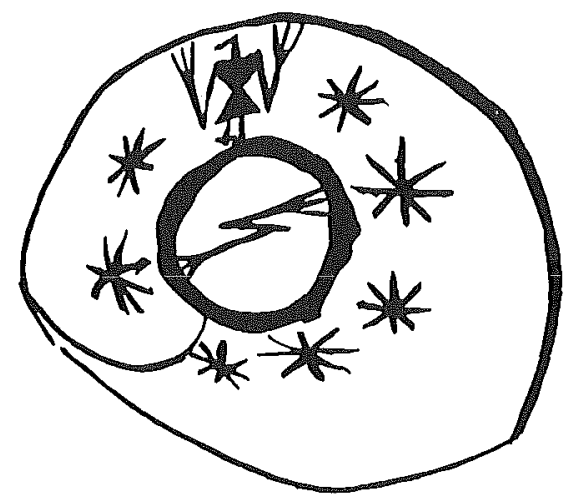

Fig. 1 : Représentation graphique du motif d'un vase de la tombe 46 de Ripacandida

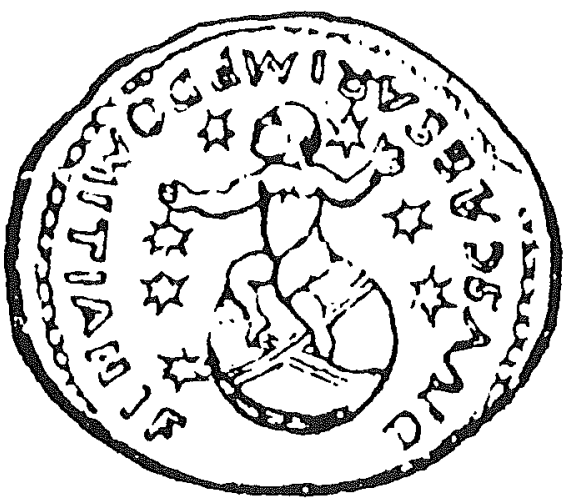

Fig. 2 : Représentation graphique d'un motif d'un denier d'or de Domitien 


\section{Les Hymnes orphiques}

Une autre catégorie de documents, toujours retrouvés dans des tombes, est représentée par des hymnes orphiques, dont le prototype est l'hymne de Derveni, exhumé partiellement consumé sur le bûcher funéraire dans une tombe d'homme enterré avec ses armes: même si tout n'est pas encore clair dans ce texte fameux (en cours de publication) sur lequel grammatici certant, il apporte bien des informations sur le courant orphique à la fin du $\mathrm{IV}^{\mathrm{e}}$ siècle (la tombe est heureusement datée avec précision), d'autant que le texte en vers est accompagné d'un Commentaire, un peu verbeux, mais d'un rare intérêt : il renseigne sur les formes cultuelles des dévots orphiques, prophéties, offrandes, sacrifices (notamment d'abord sacrifices aux Euménides, qui apparaissent comme régulatrices de la communauté ${ }^{5}$ )... Il insiste sur l'allégorie comme moyen constant de cryptage du texte sacré (Commentaire, 9).

\section{La personnalité du Zeus orphique}

Zeus règne sur l'hymne proprement dit, comme il règne sur l'univers. On peut d'abord dégager son exceptionnelle personnalité : - Zeus est le nom donné par les hommes à Moira qui existait avant que Zeus ne fût nommé et qui est la pensée divine; - Zeus est le souffle qui donne vie à tout ce qui est. Il est en même temps air et pensée; - Zeus est à la fois mâle et femelle. Il s'identifie à trois divinités féminines: Aphrodite Ouranienne, Peitho, Harmonia, qui toutes trois sont associées à sa thorné (Commentaire, 9); - Il y a identité des plus importantes divinités féminines: Gé, Méter, Rhéa, Héra (Commentaire, 18) ${ }^{6}$.

Personnage central, qui impose à tout l'univers sa volonté, selon les oracles de Nuit, Zeus agit, avec et après Nuit, comme acteur principal d'une vaste cosmo/théogonie.

\section{La cosmo/théogonie de l'hymne}

Il n'y a d'abord que deux personnages, l'initiatrice Nuit et l'initié Zeus. Sur Nuit, le Commentaire (7) affirme qu'elle prophétise depuis la profondeur de son sanctuaire, car la profondeur de la nuit, c'est l'adyton...

Nuit révèle à Zeus ce qui va lui permettre une conquête du cosmos par paliers successifs, la difficulté étant de faire agir Zeus dès les origines, donc avant qu'il ne soit Zeus le troisième souverain. Cela nécessite l'intervention d'un

5 Le rôle de surveillance de l'ordre cosmique dévolu aux Euménides est ancien. $C f$. notamment HÉrACLITE, B 94 D-K : «Le soleil n'outrepassera pas ses limites; sinon les Érinyes, servantes de Diké, le dénicheront $»$.

6 Le même Commentaire (18) précise que les hymnes identifient Déméter, Rhéa, Gé, la Mère, Hestia, Deio. 
personnage primordial jusque par son nom, le démon Protogonos, et l'utilisation de la technique de l'avalement ( $\kappa \alpha \tau \alpha$ ív $\omega$ ) qui autorise les allers-retours.

\section{Zeus l'avaleur}

- Zeus avale le glorieux démon Protogonos, le premier à avoir éjaculé dans l'Éther ${ }^{7}$; - Protogonos engendre Gaia et Ouranos; - Gaia et Ouranos engendrent Cronos. Ouranos est le premier des trois rois, mais il est donné aussi comme fils de Nuit; - L'élimination de Cronos permet à Zeus de venir au pouvoir en tant que troisième roi et il avale le principe de vie de Protogonos; - Dieux, fleuves, sources adhèrent à ce processus et Zeus devient le seul être.

\section{Retour au multiple}

- Zeus ramène à nouveau à la lumière tous les êtres recueillis dans son cœur saint : Aphrodite l'Ouranienne, Harmonie, Peitho; Gaia et Ouranos; Océanos et Achéloos; Lune et astres; - Puis il éprouve le besoin de couvrir sa mère.

Ce cheminement est le résultat d'une très longue évolution, qui (au moins d'après $\mathrm{P}$. Boyancé ${ }^{8}$ ) a largement tenu compte de l'évolution philosophique (Anaxagore, Empédocle, Platon, Aristote...).

Voilà donc ce que seuls les initiés peuvent entendre, ce qui est hautement

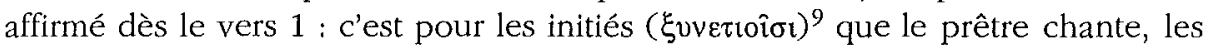
portes fermées aux profanes / impurs ( $\beta \varepsilon \dot{\beta} \eta \lambda$ o $)$.

C'est un texte étonnant d'audace, comme souvent chez les Orphiques. Il dépasse la distinction homme / femme, Zeus étant l'un et l'autre à la fois; il assimile les Grandes Déesses-Mères les unes aux autres; il construit et dissout toutes les contradictions. On comprend qu'il était urgent de joindre un Commentaire...

La litanie de la création se déroule donc au cours d'un temps primordial où n'était antérieure à Zeus que la pensée divine, Moira. Ici, pas de petites recettes d'outre-tombe permettant de se diriger lors du passage d'une vie à l'autre, mais une gigantesque évocation d'un Zeus suprême, qui ne peut cependant réussir que parce qu'il est muni des oracles de Nuit, forme et force primordiales. Il n'est pas possible que ne soit pas sauvé celui à qui de tels secrets sont révélés.

Que cette théogonie soit très différente de celle d'Hésiode est évident, mais ne fait pas vraiment problème, car on sait que les théogonies grecques, héritées

7 Je m'écarte de la traduction du vers 14 par L. BRISSON, in $R H R, 202$ (1985), p. 418 : «le vénérable, il l'avala, celui qui le premier s'élança hors de (ou dans) l'Éther ». Il y a à la fois mouvement et saillie. $C f$, dans le même sens que moi, mais peut-être plus hypothétique, R. LAMBERTON, Working edition... revised 1993, col. 9) : « He swallowed the genitals, who first impregnated (?) the ether ".

8 Remarques sur le papyrus de Derveni, in REG (1974), p. 91 sq.

9 Le Commentaire (2) les appelle mystes. 
de l'Orient, ont revêtu, au cours des siècles, bien des formes dont nos commentateurs ne démêlent l'écheveau qu'à grand-peine.

Cet hymne introduit dans des spéculations d'un très haut niveau intellectuel, fruit de siècles de «composition». La pensée centrale est celle d'un monisme rigoureusement absolu qui réduit à l'unité toutes les forces spirituelles du cosmos. Désormais on n'est plus dans un monde obéissant à une logique vulgaire. Le principe unificateur abstrait est mâle et femelle à la fois.

La sexualité est partout présente, surtout la masculine. Le mot thorné, «semence jaillissante », est bien représenté dans ce texte (comme dans une

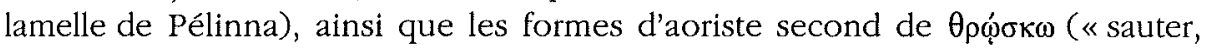
féconder, jaillir »). C'est grâce à sa thorné que Zeus conçoit Aphrodite Oura-

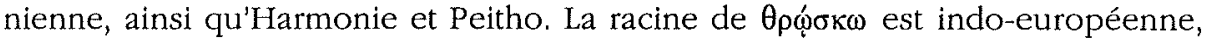
mais, à côté du grec, ne sont connues que des formes celtiques: m. irlandais dar, «saillir» et der, « jeune fille », gallois derig, « en rut ». On n'oublie pas non plus que, après avoir conçu tout l'univers, «Zeus éprouva le désir de s'unir d'amour à sa Mère »: audacieuse affirmation, aux innombrables connotations, d'un inceste primordial qui rappelle les croyances des premières sociétés humaines.

L'auteur ne peut, pour autant, ignorer les réalités mythiques essentielles : les trois générations divines, Aphrodite la Céleste et ses compagnes, Océan et Achéloos... (tous noms qui figurent aussi chez Hésiode). Il les assume, lui qui n'est pas encore né ${ }^{10}$, en les avalant, concept terrifiant qui apparaît deux fois dans ce texte de 47 hexamètres ${ }^{11}$. Il faut insister sur l'expression d'un vrai hénothéisme, lorsque sont identifiées les Grandes déesses (Commentaire 18).

On a donc ici un mixte de mythes et de personnages mythiques anciens d'une part et de l'autre de spéculations mystiques sur la personne de Zeus, sur le daimon Premier-Né (Protogonos), qui empruntent beaucoup à l'idéalisme philosophique du $\mathrm{IV}^{\mathrm{e}}$ siècle finissant. Ce Zeus est un personnage essentiel de l'orphisme primitif et il apparaît sous la double figure qu'on lui connaît sans doute depuis la Crète et les temps héroïques : enfant divin s'unissant d'amour à sa Mère, et dieu adulte de la souveraineté, détenteur de toutes les puissances.

Dans ce poème l'accent est uniquement mis sur ce Zeus mystico-philosophique. Pas d'allusion au mythe essentiel de Zagreus, à l'enfant dévoré par les Titans. Pas d'allusions dionysiaques, alors que la confusion était fréquente ${ }^{12}$. Mais un discours qui témoigne des étapes d'une prise de possession du monde par un dieu unique qui doit seulement à Nuit de pouvoir actualiser toutes les

10 Ce qui permet de répondre aux interrogations de l'édition de la Théogonie (CUF), p. 49, n. 1.

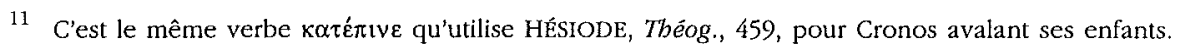

12 Cf. HDT, II, 81 : « les cultes dits orphiques et bachiques »; EUR., Hipp., 952-954 : « docile au règne d'Orphée, fais le bacchant». 
puissances qu'il porte en lui. Que risquait donc pour l'éternité un myste qui se confiait totalement à lui ?

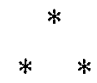

Pour tenter de saisir les rapports entre religion civique et sectes, nous avons évoqué deux catégories de documents, typiques des formes cultuelles étrangères à la cité, mais qui se développent à ses marges, en étroite connexion (dans la plupart des cas, les dieux adorés sont les mêmes) : tablettes vouées à Perséphone (mais que l'on a longtemps cru orphiques ou orphico-pythagoriciennes) et hymnes orphiques.

D'autres exemples auraient pu être analysés. Et surtout celui de Dionysos, qui n'est apparu ici qu'au détour d'une lamelle d'or, mais qui est bien le plus attractif de tous les dieux. Attractif parce ses fidèles ont une nature plus populaire que les touchants adorateurs de Perséphone et a fortioni que les sectateurs orphiques. Attractif aussi parce qu'il est partout, en ville, au théâtre, surtout dans les campagnes et les halliers qu'il parcourt avec ses Satyres et ses Bacchantes brandissant le thyrse et criant l'Evobé. Attractif enfin parce que ce dieu de la joie, des femmes, des forêts bruissant du «boucan sacré », ami de toutes les inversions sexuelles ou civiques, évite les rigueurs excessives qui détruiraient la communauté et fait de la fête une halte de liesse et d'allégresse dans la dure monotonie des travaux et des jours. Il est double, participant des plus grandes liturgies officielles, telles les Dionysies, mais recevant aussi dans des cénacles limités des fidèles qui trouvent, dans ce dieu des énergies vitales et sexuelles, un maître pour mieux participer aux allégresses du retour des saisons, des liturgies du vin et de la solidarité humaine. Ceux qui sont devenus bacchants, bebacbeuménoi forment de petites sociétés secrètes dont la force est immense dans toute la grécité. Que l'on songe aux Bacchanales de l'Italie méridionale qui inquiétèrent le Sénat de Rome au point qu'il fit massacrer des dizaines de milliers de sectateurs en 186.

Ces rites secrets, ces textes secrets, ces sociétés secrètes, nous les comprenons quelque peu. S'ils remontent certainement au deuxième millénaire, ils connaissent aux temps classiques et hellénistiques un élan remarquable qui ajoute sa contribution au miracle grec, par un équilibre qu'ils établissent avec la religion civique. Très voisins d'elle, ils n'en sont pas moins totalement autres.

Pierre LÉvÊQUE

Université de Franche-Comté

$30-32$, rue Mégevand

$\mathrm{F}-25030$ BESANÇON 